\title{
ON THE SURJECTIVITY OF LINEAR TRANSFORMATIONS
}

\author{
M. DAMLAKHI and V. ANANDAM \\ Department of Mathematics \\ Kıng Saud University \\ P O Box 2455, Riyadh 11451, SAUDI ARABIA
}

(Received March 2, 1993 and in revised form February 11, 1993)

\begin{abstract}
Let $B$ be a reflexive Banach space, $X$ a locally convex space and $T: B \rightarrow X$ (not necessarily bounded) linear transformation A necessary and sufficient condition is obtained so that for a given $v \in X$ there is a solution for the equation $T u=v$ This result is used to discuss the existence of an $L^{p}$-weak solution of $D u=v$ where $D$ is a differential operator with smooth coefficients and $v \in L^{p}$
\end{abstract}

( KEY WORDS AND PHRASES: Admissible linear operators, $L^{p}$-functions, harmonic functions 1991 AMS SUBJECT CLASSIFICATION CODES: 47F05, 47B38

\section{INTRODUCTION}

Let $T$ be a (not necessarily bounded) linear operator from a reflexive Banach space $B$ into a locally convex space $X$ We obtain a necessary and sufficient condition for the existence of a solution $u \in B$ to the equation $T u=v$, when $v \in X$ is known

In this context the following question arises naturally Let $\Omega$ be an open set in $\mathbb{R}^{n}$ and $D$ a differential operator of order $m$ with $c^{m}$-coefficients in $\Omega$ Given $v \in L^{p}(\Omega)$ does $D u=v$ have a weak solution $u \in L^{p}(\Omega)$ ?

When $p=2, \Omega$ is a bounded domain and $D$ has constant coefficients, L Hormander (to see Corollary 1 14, M Schechter [1]) has proved that $D u=v$ has always a weak solution The proof depends heavily on Hilbert space techniques as applied to $L^{2}(\Omega)$ Our investigation here is around the form the above result of Hormander takes when only Banach space methods are available as in $L^{p}(\Omega)$

\section{ADMISSIBLE LINEAR OPERATORS}

Let $B$ be a Banach space and $X$ be a locally convex space. Let $B^{\prime}$ and $X^{\prime}$ denote the algebraic duals of $B$ and $X, B^{*}$ and $X^{*}$ denote their topological duals.

Given $T: B \rightarrow X$, a linear operator not necessarily bounded, define the linear operator $T^{*}: X^{\prime} \rightarrow B^{\prime}$ as follows For $f \in X^{\prime}$ and $x \in B, T^{*} f(x)=\left\langle x, T^{*} f\right\rangle=\langle T x, f\rangle$

LEMMA 1. Let $B$ be a reflexive Banach space and $X$ be a locally convex space $T: B \rightarrow X$ is a linear operator, not necessarily bounded Suppose that there exists a subspace $H \subset X^{\prime}$ such that $T^{*}(H) \subset B^{*} \quad$ Then given $v \in X$, there exists $u \in B,\|u\| \leq c$ such that $\langle T u, f\rangle=\langle v, f\rangle$ for every $f \in H$ if and only if $|\langle v, f\rangle| \leq c\left\|T^{*} f\right\|$

PROOF. Let $\langle T u, f\rangle=\langle v, f\rangle$ with $\|u\| \leq c$ and $f \in H \quad$ Then $|\langle v, f\rangle|=\left|\left\langle u, T^{*} f\right\rangle\right| \leq\|u\|$ $\left\|T^{*} f\right\| \leq c\left\|T^{*} f\right\|$

Conversely, define the linear functional $S$ on the subspace $T^{*}(H)$ so that, for $g \in T^{*}(H)$, $S g=\langle v, f\rangle$ where $g=T^{*} f$ for some $f \in H$

$S$ is well-defined, for, if $g=T^{*} f_{1}$, for some other $f_{1} \in H$, then $\left|\langle v, f\rangle-\left\langle v, f_{1}\right\rangle\right|=\left|\left\langle v, f-f_{1}\right\rangle\right| \leq$ $c\left\|T^{*}\left(f-f_{1}\right)\right\|=0$ 
It is clear that $S$ is a bounded linear functional on the subspace $T^{*}(H) \subset B^{*}$ with $\|S\| \leq c$ and hence by Hahn-Banach theorem extends as a bounded linear functional on $B^{*}$, preserving the norm

This implies, since $B$ is reflexive, that there exists $u \in B$ such that for every $h \in B^{*},\langle u, h\rangle=S h$ and $\|u\|=\|S\|=c$

In particular, if $h=T^{*} f, f \in H$, we have $\left\langle u, T^{*} f\right\rangle=S\left(T^{*} f\right)=\langle v, f\rangle$

Thus, for any $f \in H,\langle v, f\rangle=\left\langle u, T^{\star} f\right\rangle=\langle T u, f\rangle$

This completes the proof of the lemma

REMARK 2.1. The above lemma is inspired from section 16 of M Schechter [1] where the existence of a weak solution of a differential operator in the Hilbert space $L^{2}(\Omega)$ is investigated

DEFINITION 2.1. Let $B$ be a Banach space and $X$ be a locally convex space A linear operator $T \cdot B \rightarrow X$, is said to be admissible if there exists a weak dense subspace $M \subset X^{*}$ such that $T^{*}(M) \subset B^{*}$

PROPOSITION 2.1. Let $B$ be a Banach space and $X$ be a Fréchet space Let $T: B \rightarrow X$ be a linear operator Then $T$ is continuous if and only if $T$ is admissible

PROOF. If $T$ is continuous, then for any $f \in X^{*}$ clearly $T^{*} f \in B^{*}$ and hence $T$ is admissible

Conversely, let $T$ be admissible with $T^{*}(M) \subset B^{*}$ where $M$ is a weak ${ }^{*}$-dense subspace of $X^{*}$ We will prove that $T$ is continuous by showing that $T$ is closed (W Rudin [2], p 50)

Let $x_{n} \in B$ be a sequence such that $\lim _{n} x_{n}=x$ and $\lim _{n} T x_{n}=y$ Then for any $f \in M$, $\left\langle x_{n}, T^{*} f\right\rangle=\left\langle T x_{n}, f\right\rangle$

Taking limits $\left\langle x, T^{*} f\right\rangle=\langle y, f\rangle$ which implies that $\langle T x, f\rangle=\langle y, f\rangle$ for every $f \in M$ and consequently $\langle T x, h\rangle=\langle y, h\rangle$ for every $h \in X^{*}$, since $M$ is $W^{*}$-dense in $X^{*}$.

This implies that $T x=y$ since $X^{*}$ separates $X$, that is, $T$ is closed

THEOREM 2.1. Let $B$ be a reflexive Banach space and $X$ be a locally convex space Let $T: B \rightarrow X$ be an admissible linear operator with $T^{*}(M) \subset B^{*}$ Then, for any given $v \in X$ there exists $u \in B$ such that $\|u\| \leq c$ and $T u=v$ if and only if $|\langle v, f\rangle| \leq c\left\|T^{*} f\right\|$ for every $f \in M$

PROOF. In view of Lemma 1 (where we take $H=M$ ), it is enough to prove that the condition $\langle T u, f\rangle=\langle v, f\rangle$ for every $f \in M$ is equivalent to the fact that $T u=v$

Now, the condition above is equivalent to the fact $\langle T u, h\rangle=\langle v, h\rangle$ for every $h \in X^{*}$, since $M$ is dense in $X^{*}$ with its $W^{*}$-topology

Since $X^{*}$ separates points on the locally convex space $X$, the latter condition is equivalent to the fact $T u=v$

\section{WEAK SOLUTIONS IN $L^{P}(\Omega)$}

Let $\Omega$ be a domain in $\mathbb{R}^{n}, n \geq 1$. Let $A=\sum_{|k| \leq m} a_{k}(x) D^{k}$ be a differential operator of order $m$, with $a_{k}(x) \in c^{m}(\Omega)$ Let $A^{*}$ denote the adjoint operator Let $1<p \leq \infty$ and $\frac{1}{p}+\frac{1}{q}=1$

THEOREM 3.1. With the above assumptions on $A$ and $p$, let $f \in L^{p}(\Omega)$ be given Then there exists a weak solution of $A u=f, u \in L^{p}(\Omega)$ and $\|u\|_{p} \leq c$ if and only if $|\langle\phi, f\rangle| \leq c\left\|A^{*} \phi\right\|_{q}$ for all $\phi \in C_{0}^{\infty}(\Omega)$

PROOF. Suppose $f \in L^{p}$ and $A u=f$ has a weak solution $u \in L^{p},\|u\| \leq c$

Define, for $\phi \in c_{0}^{\infty}(\Omega)$ and $g \in L^{p}(\Omega),\langle\phi, g\rangle=\int_{\Omega} \bar{g}(x) \phi(x) d x$

Then, $|\langle\phi, f\rangle|=|\langle\phi, A u\rangle|=\left|\left\langle A^{*} \phi, u\right\rangle\right| \leq\|u\|_{p}\left\|A^{*} \phi\right\|_{q} \leq c\left\|A^{*} \phi\right\|_{q}$

Conversely, define the linear functional $S$ on the subspace $A^{*}\left(c_{0}^{\infty}(\Omega)\right)$ such that $S\left(A^{*} \phi\right)=\langle\phi, f\rangle=$ $\int_{\Omega} \bar{f} \phi d x$

Then, as in Lemma $1, S$ is a well-defined linear functional on $A^{*}\left(c_{0}^{\infty}(\Omega)\right) \subset L^{q}(\Omega)$ with $\|S\| \leq c$ and hence extends as a continuous linear functional on $L^{q}(\Omega)$, so that there exists $u \in L^{p}(\Omega)$ satisfying the condition $S(v)=\langle v, u\rangle$ for all $v \in A^{*}\left(c_{0}^{\infty}(\Omega)\right)$ and $\|u\|_{p}=\|S\| \leq c$ 
In particular, for any $\phi \in c_{0}^{\times}(\Omega),\langle\phi, f\rangle=S\left(A^{*} \phi\right)=\left\langle A^{*} \phi, u\right\rangle=\langle\phi, A u\rangle \quad$ Hence $u$ is a weak solution of $A u=f$

THEOREM 3.2. Let $f \in L_{l o c}^{1}(\Omega)$ Then there exists a bounded weak solution $u$ of the equation $A u=f$ if and only if $\left|\int_{\Omega} \bar{f}(x) \phi(x) d x\right| \leq C\left\|A^{*} \phi\right\|_{1}$, for every $\phi \in c_{0}{ }^{\times}(\Omega)$

PROOF. In view of the above theorem, we will give here only a few details of the proof

On $A^{*}\left(c_{0}{ }^{\lambda}(\Omega)\right)$, considered as a subspace of $L^{1}(\Omega)$, define the linear functional $S$ such that $S\left(A^{*} \phi\right)=\langle\phi, f\rangle=\int \bar{f} \phi d x$ Then $S$ extends as a bounded linear functional on $L^{1}(\Omega)$ so that there exists $u \in L^{\times}(\Omega)$ such that $S g=\langle g, u\rangle$ for every $g \in L^{1}(\Omega)$

This leads to the fact that $u$ is a weak solution of $A u=f$

In the context of the above theorem where we were looking for a bounded weak solution of a differential equation, the following proposition concerning the bounded solutions of the Laplacian in $\mathbb{R}^{n}$ is of interest

PROPOSITION 3.1. Let $f \in C_{0}^{\times \times}\left(\mathbb{R}^{n}\right)$, having compact support $K$, be given in $\mathbb{R}^{n}$ Then, if $n \geq 3$, there always exists a bounded $u \in c^{\times} \cdot\left(\mathbb{R}^{n}\right)$ such that $\Delta u=f$, if $n=1$ or 2 , such a bounded $c^{\times}$solution exists if and only if $\int_{K} f(x) d x=0$

PROOF. Since $\triangle$ is an elliptic differential operator with constant coefficients, there always exists some $u \in c^{\times}\left(\mathbb{R}^{n}\right)$ such that $\Delta u=f$ Here we are looking for a bounded function $u$ in $c^{(x)}\left(\mathbb{R}^{n}\right)$

Let

$$
E_{n}(x)= \begin{cases}|x| & \text { if } n=1 \\ \log |x| & \text { if } n=2 \\ -\frac{1}{|x|^{n-2}} & \text { if } n>3\end{cases}
$$

Now, using the results in [2], we can show that for a fixed $y \in K$ and any $x \in K^{c}$,

$$
u(x)=\left(\int f(x) d x\right) \beta_{n} E_{n}(x-y)+l(x)
$$

where $l(x)$ is a bounded harmonic function in $K^{c}$ if $n \geq 2$ (and affine bounded if $n=1$ )

Here $\beta_{1}=\frac{1}{2}, \beta_{2}=\frac{1}{2 \pi}$ and $\beta_{n}=\frac{1}{(n-2) \alpha_{n}}$ if $n \geq 3, \alpha_{n}$ being the measure of the unit sphere in $\mathbb{R}^{n}$

Consequently, using the fact that $E_{n}(x)$ is bounded in a neighborhood of the point at infinity if and only if $n \geq 3$, we arrive at the conclusion of the proposition

NOTE. Since a bounded harmonic function outside a compact set in $\mathbb{R}^{n}, n \geq 2$, tends to a limit at infinity, if $u$ is a bounded solution of $\triangle u=f \in c_{0}^{\infty}$, we can choose $u_{0} \in c^{\infty}\left(\mathbb{R}^{n}\right)$ so that $u_{0}$ tends to 0 at infinity and satisfies the condition $\triangle u_{0}=f$ In this case, $u_{0}$ is unique

\section{SURJECTIVITY ON THE SOBOLEV SPACES}

We conclude this article with a remark on the solutions of a differential operator on the Sobolev spaces $H^{s}\left(\mathbb{R}^{n}\right)$

We make use of the following properties.

i) For each real $s, H^{s}\left(\mathbb{R}^{n}\right)$ is a Hilbert space such that $H^{s} \subset H^{t}$ if $t \leq s$

ii) $H^{s}$ is the completion of $c_{0}^{\infty}$ in the norm $\|\cdot\|_{H^{s}}$

iii) For any $s, H^{-s}$ represents the topological dual of $H^{s}$

iv) If $s>\frac{n}{2}+k$ where $k$ is a nonnegative integer, then $H^{s} \subset c^{k}$

v) If $A$ is a differential operator of order $m$ with $c^{m}$-coefficients, $A^{*}\left(c_{0}^{\infty}\right) \subset L^{2} \subset H^{s}$ for any $s \geq 0$

vi) If $A$ is a differential operator of order $m$ with $c^{\infty}$-coefficients, $A^{*}\left(c_{0}^{\infty}\right) \subset H^{s}$ for every real $s$

Then, with arguments similar to those utilized to prove some of the earlier results, we obtain 
THEOREM 4.1. Let $T$ be a distribution in $\mathbb{R}^{n}, n \geq 1$ Suppose that $A$ is a differential operator of order $m$ satisfying one of the following two sets of assumptions

a) $A$ has $c^{m}$-coefficients and $s \geq 0$

b) $A$ has $c^{\prime x}$-coefficients and $s$ is any real number

Then $T=A u$ in the sense of distribution, for some $u \in H^{*}$, if and only if $|T(\phi)| \leq c\left\|A^{*} \phi\right\|_{11}$, for all $\phi \in c_{0}^{\times}\left(\mathbb{R}^{n}\right)$

REMARK 4.1. Let $A$ be a differential operator of order $m$ with coefficients either constants or from the Schwartz's space (i e rapidly decreasing $c^{(x)}$-functions) Then if $|T(\phi)| \leq c\left\|A^{*} \phi\right\|_{H}$. for all $\phi \in c_{0}^{\times}$, we have as in the above theorem, $T=A u, u \in H^{*}$

But, in this special case, $T \in H^{s} m$ and consequently, if $s>\frac{n}{2}+m$, then $T=A u$ in the classical sense i e $u$ is a strong solution of the differential equation

$H^{k}(\Omega)$-spaces Let now $\Omega$ be an open set in $\mathbb{R}^{n}, n \geq 1$ Recall that for any positive integer $k$, $H_{0}^{k}(\Omega)$ is defined as the closure of $c_{0}^{(x)}(\Omega)$ in $H^{k}(\Omega)$ For any $v \in L^{2}(\Omega)$, define $\|v\|_{-k}=\sup _{u \in H_{0}^{k}(\Omega)} \frac{\left|\left\langle v u_{0}\right\rangle\right|}{\|\|_{H^{k}(\Omega)}}$ Then, if $H^{k}(\Omega)$ denotes the completion of $L^{2}(\Omega)$ in the norm $\|\cdot\|_{-k}, H^{-k}(\Omega)$ is the topological dual of $H_{0}^{k}(\Omega)$ for any integer $k \geq 0$ (see Al-Gwaiz [4], p 191)

With this background, we can state an analogue of Theorem 41 as follows

THEOREM 4.2. Let $T$ be a distribution in an open set $\Omega$ in $\mathbb{R}^{n}, n \geq 1$ Suppose $A$ is a linear differential operator with $c^{\infty}(\Omega)$-coefficients Then, for any integer $k \geq 0$, there exists $u \in H^{-k}(\Omega)$ such that $A u=T$ if and only if $|T \phi| \leq c\left\|A^{*} \phi\right\|_{H^{k}(\Omega)}$ for all $\phi \in c_{0}^{\infty}(\Omega)$

\section{REFERENCES}

[1] SCHECHTER, M , Modern Methods in Partial Differentıal Equatıons, McGraw-Hill Inc, 1977

[2] RUDIN, W , Functional Analysis, McGraw-Hill Inc , 1973.

[3] ANANDAM, V., Espaces harmoniques sans potentiel positif, Ann. Inst. Fourier 22 (1972), 97-160

[4] AL-GWAIZ, M, Theory of Distributions, Marcel Deker Inc., New York, 1992 


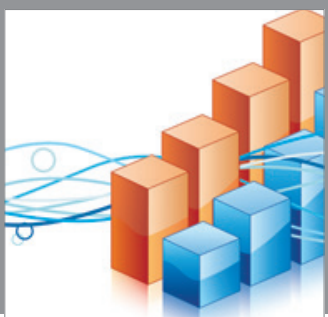

Advances in

Operations Research

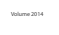

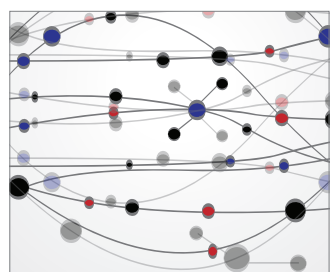

\section{The Scientific} World Journal
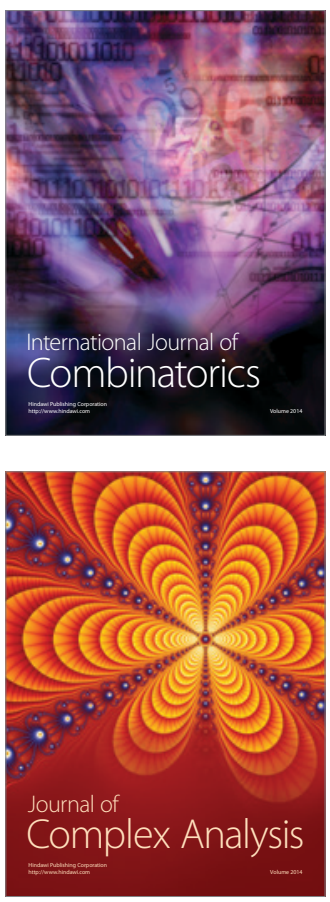

International Journal of

Mathematics and

Mathematical

Sciences
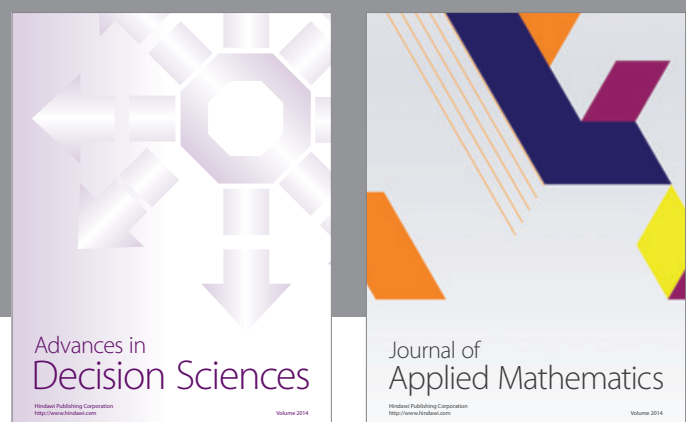

Journal of

Applied Mathematics
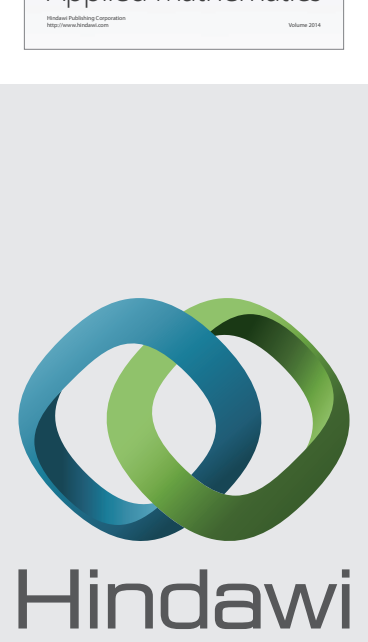

Submit your manuscripts at http://www.hindawi.com
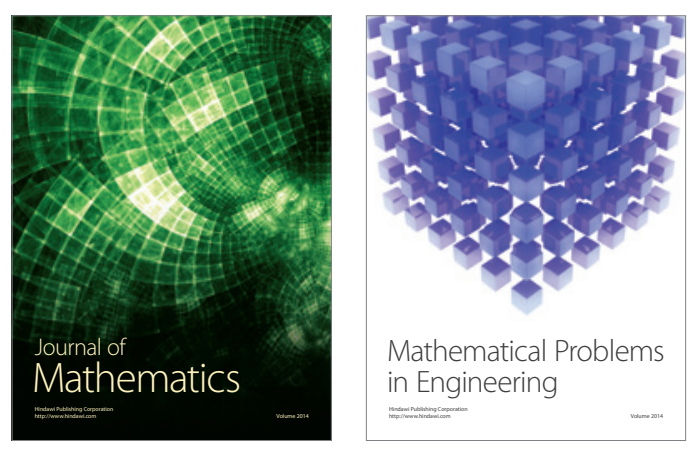

Mathematical Problems in Engineering
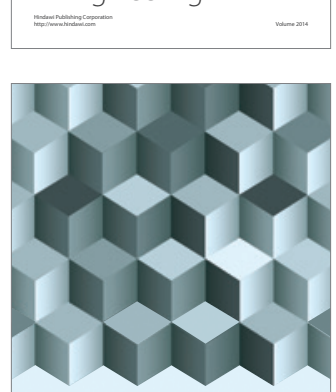

Journal of

Function Spaces
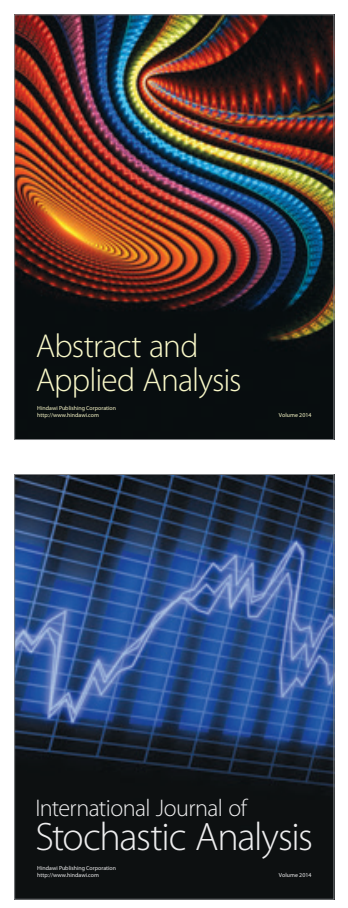

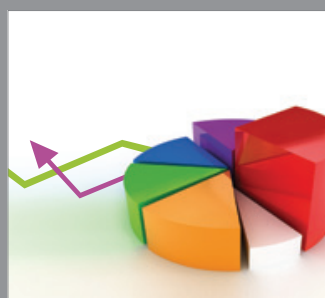

ournal of

Probability and Statistics

Promensencen
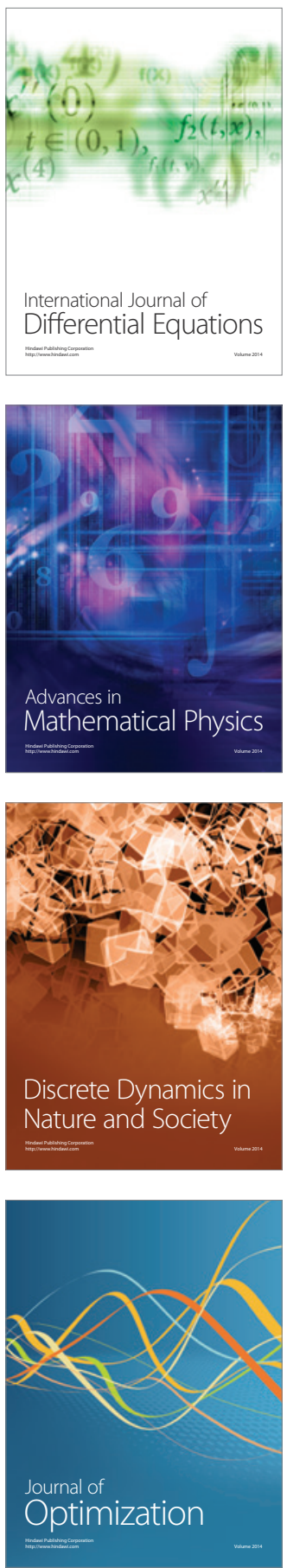